\title{
Suitability of a probiotic Lactobacillus paracasei strain as a starter culture in olive fermentation and development of the innovative patented product "probiotic table olives"
}

\section{Angelo Sisto and Paola Lavermicocca*}

Institute of Sciences of Food Production, National Research Council, Bari, Italy

\section{Edited by:}

Aldo Corsetti, University of Teramo Italy

\section{Reviewed by:}

Maria Guadalupe Vizoso Pinto, Max von Pettenkofer Institute of the Ludwig Maximilian University of Munich, Germany

Francisco Noé Arroyo López, Instituto de la Grasa (Consejo Superior de Investigaciones Cientificas), Spain Luca Settanni, Università degli Studi di Palermo, Italy

Svetoslav Todorov, University of Sao

Paulo, Brazil

\section{*Correspondence:}

Paola Lavermicocca, Institute of Sciences of Food Production, National Research Council, Via Amendola 122/O, 70126 Bari, Italy. e-mail: paola.lavermicocca@ispa.cnr.it
Probiotic bacteria are generally available for consumers as concentrated preparations or incorporated in milk-based foods. Due to an increased interest of the market for probiotic foods as well as to meet a demand of industry for innovation, a new kind of probiotic food has been developed using table olives as a carrier. Green table olives, produced according to the Spanish-style, are obtained by a fermentation which can be carried out by spontaneous microflora, even if the use of starter cultures is desirable to obtain a more controlled process. In this regard, the selected strain Lactobacillus paracasei IMPC 2.1 of human origin was used in the dual role of starter and probiotic culture, and here we describe the different aspects which have been evaluated and solved to utilize that strain for the development of a new table olive-based probiotic food. These aspects include selection of the strain on the basis of its probiotic properties, molecular characterization, compatibility with the carrier food, and efficacy as starter. The final product meets commercial and functional requirements throughout its shelf-life.

Keywords: functional food, probiotic food, lactic acid bacteria, olive surface
Green table olives are obtained from fruits of the cultivated olive tree (Olea europaea L.) harvested during the ripening period when the final size is reached but the color is still green. After harvesting, olives contain phenols and oleuropein which makes the fruits unpalatable due to their bitter taste. According to the most well-known method of processing (de Castro et al., 2002), i.e., the "Spanish-style," bitterness in the fruits can be almost completely eliminated by alkaline hydrolysis of oleuropein (Ciafardini etal., 1994) treating olives with a sodium hydroxide solution (lye). The next step is a washing process to remove the excess alkali. Then, olive fruits are submerged into a brine (aqueous solution of up to $12-13 \% \mathrm{NaCl}$, w/v) where a lactic acid fermentation takes place and in which a rapid initiation of growth of lactic acid bacteria (LAB) is required to avoid product spoilage by undesirable bacteria. Spontaneous fermentation of Spanishstyle preparations of green olives includes three different phases of microbial succession. The first phase is dominated by Gramnegative bacteria (Enterobacteriaceae). These bacteria decrease in the second phase characterized by a progressive growth of LAB belonging to Leuconostoc and Pediococcus genera. The third phase is characterized by the relevant proliferation of lactobacilli mainly of the species Lactobacillus plantarum and L. pentosus, but also L. rhamnosus and L. paracasei have been identified (Van den Berg et al., 1993). Lactic acid fermentation performed by these bacteria produces mainly lactic acid causing a $\mathrm{pH}$ decrease. At the end of the process, when fermentation is complete, the final product is stable and can be stored in brine under appropriate conditions $(\mathrm{NaCl}>5 \% ; \mathrm{pH}<3.5)$ without refrigeration. Even if the fermentation of table olives can be carried out by the spontaneous microbiota, the use of starter cultures is an advisable practice. Generally, the starter is a selected strain which belong to a species component of the spontaneous microbiota. At the end of fermentation, table olives are a natural product still hosting on their surface a viable microbiota (Lavermicocca et al., 1998).

The evidence that the surface of olive fruits is colonized by LAB paved the way for the use of table olives as a biological carrier for beneficial strains such as probiotics (Lavermicocca et al., 2003, 2005). The recent interest for the beneficial health effects that foods may have beyond their basic nutritional value has recently led to a worldwide increase in consumer interest for functional products, including probiotic foods, and to a huge increase in their commercial value. Probiotic products contain selected microbial strains which - if they reach the gastrointestinal (GI) tract in viable form and adequate amounts - can improve GI function, and thus, the health of the host (FAO/WHO, 2001). Species of the genera Bifidobacterium and Lactobacillus are widely marketed for human consumption as probiotics in concentrated preparations available as capsules, powders, or liquid products, or else incorporated into milk-based functional foods. But the market of functional food industries is currently interested in new non-dairy foods which could have the potential to attract more consumers to functional products allowing also a more regular intake of probiotics. In this regard, the cited studies (Lavermicocca et al., 2005) 
have demonstrated that table olives are an excellent alternative food carrier suitable for delivering probiotics to humans. Since a probiotic strain has to be administered in adequate amount to exert its beneficial effect, it should be also able to survive and firmly establish itself on table olives competing with the other microorganisms and in particular with the starter strain. Therefore, the selection and the use of a probiotic strain even able to act as a starter to pilot the fermentation would be highly advisable.

In this review we highlight the different aspects which have been evaluated and solved to develop a new table olive-based probiotic food with the Lactobacillus paracasei strain IMPC 2.1, of human origin, playing the dual role of starter and probiotic culture. The final product is an innovative patented probiotic food which meets all commercial and functional requirements throughout its shelflife of several months and received the marketing authorization from the Italian Ministry of Health.

\section{CHARACTERIZATION AND SELECTION OF THE PROBIOTIC STRAIN}

The selection of a probiotic strain requires the evaluation of a number of characteristics which enable the strain to cause beneficial effects after its administration. Probiotics are administered orally and need to survive through the complex physical-chemical environment of the GI tract. Since probiotic bacteria only transiently colonize the intestinal tract, large populations have to be ingested on a daily basis to obtain beneficial effects (Reid et al., 2001,2003 ) and $10^{9}$ viable cells per serving is the minimal dose recommended (FAO/WHO, 2001, 2002; Maukonen et al., 2006; Health Canada, 2009). Each new proposed probiotic strain must be characterized since it is widely recognized that probiotic features are strictly strain-specific and cannot be considered as typical characteristics of a bacterial species. The ability of a strain to survive passage through the GI tract is generally the first evaluated aspect in the selection of a probiotic strain and can be mainly attributed to its acid and bile tolerance. Moreover, the adhesion to intestinal mucosa is relevant as it seems to be related to the ability of a bacterial strain to persist in the gut (Servin and Coconnier, 2003). A number of in vitro tests of simulated digestion and adhesion have been proposed and used to preliminarily evaluate the ability of a probiotic strain to survive the passage through and to colonize the GI tract; however, in a next phase these characteristics have to be ascertained by in vivo experiments. In vivo tests are very important also because strain survival partially depends on the potential protective action of the associated carrier food which is due in some way to its chemical composition. In fact, Charalampopoulos et al. (2003) demonstrated that the presence of wheat, barley, and malt extracts increased the viability of L. plantarum, L. acidophilus, and L. reuteri strains under acidic conditions simulating the gastric tract. The authors attributed this result to the composition of cereal extracts and mainly to the presence of soluble sugars. The protective effect of metabolizable sugars was confirmed by Corcoran et al. (2005) who found that the presence of glucose and fructose highly increased the survival of some strains of L. rhamnosus, L. gasseri, and L. paracasei treated with simulated gastric juice and that viability varied among species. These results demonstrate that, when the final aim is the formulation of a new probiotic food, the interaction between the probiotic strain and the carrier food has to be taken into account in evaluating the compatibility between the strain and the carrier food as well as the potential protective action of the carrier under GI conditions.

In the case of the selection of L. paracasei IMPC 2.1 its characteristics were compared with those of three strains of L. rhamnosus (including the well-characterized probiotic strain GG), five strains of L. plantarum, and one strain of the species L. paracasei, L. alimentarius, and L. fermentum. It was preliminarily ascertained by in vitro tests its resistance to simulated gastric juice and to bile salts as well as its adhesion to pig mucus. Strain IMPC 2.1 was also demonstrated to be strongly adhesive to enterocyte-like Caco-2 cells (Lavermicocca et al., 2003, 2008) as it showed a better adhesion than the probiotic strain L. rhamnosus GG, which has strong adhesive properties to human intestinal epithelial cells (Elo et al., 1991). The survival on table olives of strain IMPC 2.1 was also evaluated and compared with that of strains of the species L. rhamnosus, L. paracasei, Bifidobacterium bifidum, and B. longum. The results clearly indicated the compatibility between table olives and strain IMPC 2.1 which showed the highest viability, with more than $10^{7} \mathrm{CFU} / \mathrm{g}$, throughout the 3-month experiment; the adhesion of strain IMPC 2.1 to olive surface was also demonstrated by scanning electron microscopy (Lavermicocca et al., 2005; Figure 1). Moreover, in vitro experiments demonstrated that the presence of olive fruits had a protective effect on strain IMPC 2.1 during simulated gastric and intestinal digestion since the survival of strain IMPC 2.1 was markedly increased (Valerio et al., 2006). Although the above-described results suggested that table olives are a suitable biological carrier for delivering strain IMPC 2.1 in the GI tract, actually a definitive evidence was obtained only by an in vivo study. In fact, populations of strain IMPC 2.1 were detected in fecal samples of human volunteers fed with 10-15 olives/day carrying about $10^{9}$ to $10^{10}$ viable cells per serving of strain IMPC 2.1 for 10 days (Lavermicocca et al., 2005).

In this regard, detection of a probiotic strain within a complex microbiota, such as those of fecal or vegetable samples,

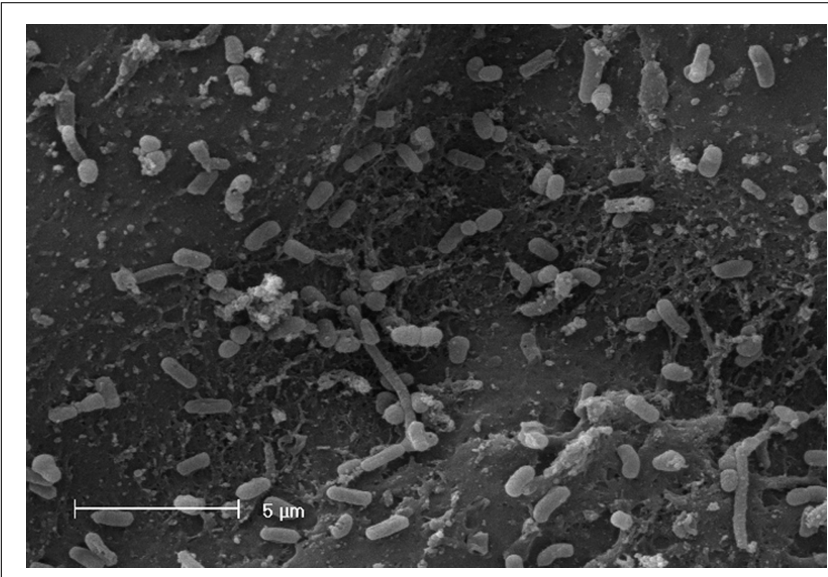

FIGURE 1 | Adhesion of Lactobacillus paracasei IMPC 2.1 cells to the surface of an olive fruit (scanning electron microscopy observation by Maria Luisa Callegari). 
requires the development of a strain-specific method of identification. The identifications of a probiotic strain at the species as well as at the strain levels are important aspects which need to be considered also because both identification and strain recognition are required to obtain the authorization, from national or international health authorities, to use a probiotic strain for human feeding. Nowadays, a strain-specific identification can be obtained using a wide range of molecular tools which also need to obtain a certain identification at the species level. L. paracase $i$ strain IMPC 2.1 was identified and characterized at molecular level by using methods such as 16S rRNA gene sequencing, REP-PCR, and the amplified fragment length polymorphism (AFLP) analysis. Moreover, based on a strain-specific AFLP molecular marker, a strain-specific PCR test was developed allowing differentiation of strain IMPC 2.1 also from other strains belonging to the same bacterial species (Sisto et al., 2009). In fact, the development of new probiotic foods requires a frequent monitoring of the probiotic strain's survival in those products within an ecosystem characterized by a complex microbiota. An approach similar to the one used for strain IMPC 2.1 could be adopted to develop easy and rapid detection methods for monitoring also other probiotic bacteria in complex microbiota, mainly when frequent evaluations are required.

\section{BENEFICIAL EFFECTS OF THE PROBIOTIC STRAIN}

Beneficial effects of probiotic bacteria are the results of the complex interactions occurring among probiotic bacteria, human host and its intestinal microbiota (Lebeer et al., 2008). Generally, the health promoting capacities of probiotic bacteria are based on one of the following, sometimes overlapping, mechanisms: (i) pathogen inhibition, (ii) restoration of microbial homeostasis through microbe-microbe interactions, (iii) enhancement of epithelial barrier function, and (iv) modulation of immune responses. Taking into account the complexity of these mechanisms it is not unexpected that each probiotic strain can determine a different response in the host. Therefore, results with one specific strain cannot be generalized and the strain-specific properties must be studied and ascertained. In the case of L. paracasei IMPC 2.1 , the ability of this strain to antagonize the growth of the foodborne human pathogen Yersinia enterocolitica was demonstrated as well as its ability to inhibit the pathogen ureolytic activity leading to the production of ammonia (Lavermicocca et al., 2008). Studies also showed that strain IMPC 2.1, administered with artichokes to volunteers suffering from constipation, gave rise to an overall positive effect on symptom profile of the participants. Moreover, strain IMPC 2.1 successfully colonized the human gut and positively influenced fecal biochemical parameters and bacteria. In fact, this probiotic strain caused the inhibition of potentially harmful bacteria (Escherichia coli and Clostridium spp.) and stimulated the growth of LAB and of lactobacilli and bifidobacteria. The human trials also confirmed that the introduction of vegetables enriched with probiotics such as artichokes, likewise olives, represents a way to achieve the target "functional diet" (Valerio et al., 2010, 2011; Riezzo et al., 2012).

Among the other mechanisms by which probiotic bacteria cause beneficial effects on the host, modulation of immune responses has been particularly studied and extensively reviewed
(Delcenserie et al., 2008; Borchers et al., 2009; Yan and Polk, 2011). Immunomodulatory properties of strain IMPC 2.1 were evaluated in two studies which highlighted its interesting characteristics. In particular, the first study (D'Arienzo et al., 2009) was carried out to analyze immunomodulatory approaches potentially useful for Celiac disease, a common food-sensitive enteropathy with an autoimmune basis caused by the lack of oral tolerance to wheat gluten and related prolamines (Maki et al., 2003). In this regard, the effects of different bacterial strains were evaluated on transgenic mice sensitive to gluten (gliadin), which represent a well-characterized model of sensitivity to food antigen useful to study in vivo the influence of probiotics on intestinal antigenspecific immune responses (Black et al., 2002; Senger et al., 2005). The results of in vivo challenge with strain IMPC 2.1 indicated its ability in enhancing the gliadin-specific production of the proinflammatory cytokine TNF- $\alpha$, thus highlighting the ability of the probiotic to exert inductive rather than suppressive effects. In a second study (D'Arienzo et al., 2011), the immunomodulatory properties of strain IMPC 2.1 were compared with those of other strains belonging to the same species $L$. paracasei. In this study, the interactions between those bacterial strains and cells of the immune system were considered, in particular evaluating the ability of the strains to cause cytokine secretion by dendritic cells, which play an essential role in both innate and adaptive immunity (Borchers etal., 2009) and are important in earliest bacterial recognition and in determining the subsequent $\mathrm{T}$ cell responses. The results revealed the different immunomodulatory properties of strains belonging to the same bacterial species. Probiotic strains IMPC 2.1 and LMG P-17806 (= F19; Crittenden et al., 2002; Ohlson et al., 2002) were characterized by a similar and intermediate ability to induce cytokine secretion. In fact, they induced a low pro-inflammatory response, not resulting in an inflammatory outcome (but enough to induce a state of host immune system alertness) and were clearly differentiated from the non-probiotic strain LMG 23554 (= YS8866441; Daniel et al., 2006) which showed a low ability to stimulate cytokine secretion. Therefore, for each new potential probiotic strain, the profiles of the cytokines whose secretion is induced should be established to define its pro- or anti-inflammatory properties and the more appropriate clinical use.

\section{SUITABILITY OF THE PROBIOTIC STRAIN AS A STARTER IN OLIVE FERMENTATION AND TECHNOLOGICAL PROPERTIES}

Although olive fermentation can be carried out by the spontaneous microflora, since the natural microflora of olives is mainly destroyed by the lye treatment that precedes the fermentation step, the use of LAB as starter cultures is an advisable practice that offers the advantage to induce a suitable development of the fermentation process allowing a faster $\mathrm{pH}$ decrease with respect to the spontaneous process. Moreover, the use of a starter favors a better standardization of the organoleptic properties of the final product. From the technological point of view, an efficient starter strain should possess some physiological characteristics including: (i) good resistance to high levels of salt as well as to extreme alkaline or acidic $\mathrm{pH}$ values, (ii) the ability to utilize completely the fermentable substrates causing a rapid $\mathrm{pH}$ decrease 
due to organic acid production, (iii) ability to compete with the spontaneous microbiota, (iv) potential inhibitory effects against spoilage (or pathogenic) microorganisms, and (v) good characteristics of survival and revitalization after storage as a freeze-dried culture. Generally, the starter microorganism is a selected strain which belong to a Lactobacillus species component of the spontaneous microbiota. In fact, the use of strains belonging mainly to $L$. plantarum/L. pentosus species successfully improved green olive processing hampering the effect of spoilage microorganisms (Garrido-Fernández et al., 1997; Sánchez et al., 2001; Panagou et al., 2003; Vega Leal-Sánchez et al., 2003). Moreover, strains of the species $L$. paracasei have been isolated from olive fermentations (Van den Berg et al., 1993) and to our knowledge, at least experimentally, a strain of $L$. paracasei from dairy origin was successfully used in green olive fermentation (Panagou et al., 2007). Probiotic strain $L$. paracasei IMPC 2.1 is of human origin, therefore, it is better adapted than strains isolated from different environments to colonize the human intestine. This strain was originally selected on the basis of its probiotic properties; then, its suitability as a starter was first studied at a laboratory level and then in an industrial plant (De Bellis et al., 2010). The study allowed to assess the persistence and the efficacy of strain IMPC 2.1 as a starter throughout the fermentation process, and to ascertain the dynamics of microbial populations associated with olive surface in industrial fermentation sets at 4 or $8 \% \mathrm{NaCl}$ and at room temperature or $4^{\circ} \mathrm{C}$. The results indicated that the probiotic strain successfully colonized the olive surface and persisted in high numbers allowing the reduction of $\mathrm{pH}$ of brines to values lower than 5.0 after 30 days and until the end of fermentation. The dynamics of microbial populations associated with olive surface and belonging to different groups indicated that olives inoculated with the probiotic did not host Enterobacteriaceae at the end of fermentation, while yeast populations were present in a low number throughout the process. The results also revealed a considerable genetic diversity of LAB species colonizing the olive surface and the effect of the probiotic strain on LAB populations. In fact, species of the Enterococcus casseliflavus group, mainly forming LAB populations before the fermentation process, were not identified in sets inoculated with the probiotic strain already at the beginning of fermentation. Moreover, in inoculated sets the number of

\section{REFERENCES}

Black, K. E., Murray, J. A., and David, C. S. (2002). HLA-DQ determines the response to exogenous wheat proteins: a model of gluten sensitivity in transgenic knockout mice. $J$. Immunol. 169, 5595-5600.

Borchers, A. T., Selmi, C., Meyers, F. J., Keen, C. L., and Gershwin, M. E. (2009). Probiotics and immunity. J. Gastroenterol. 44, 26-46.

Charalampopoulos, D., Pandiella, S. S., and Webb, C. (2003). Evaluation of the effect of malt, wheat and barley extracts on the viability of potentially probiotic lactic acid bacteria under acidic conditions. Int. J. Food Microbiol. 82, 133-141.
Ciafardini, G., Marsilio, V., Lanza, B., and Pozzi, N. (1994). Hydrolysis of oleuropein by Lactobacillus plantarum strains associated with olive fermentation. Appl. Environ. Microbiol. 60, 4142-4147.

Corcoran, B. M., Stanton, C., Fitzgerald, G. F., and Ross, R. P. (2005). Survival of probiotic lactobacilli in acidic environments is enhanced in the presence of metabolizable sugars. Appl. Environ. Microbiol. 71, 3060-3067.

Crittenden, R., Saarela, M., Mättö, J., Ouwehand, A. C., Salminen, S., Pelto, L., Vaughan, E. E., De Vos, W. M., Von Wright, A., Fonden, R., and Mattila-Sandholm, T. (2002). Lactobacillus paracasei subsp. paracasei

L. pentosus was significantly reduced during the process, while it was the dominant species from 20 days up to the end of the process in control sets. Although our results indicate that, at the end of fermentation, probiotic olives host a significant genetic diversity of $\mathrm{LAB}$ species on their surface together with probiotic strain IMCP 2.1, they are considered a natural probiotic product as they provide to the consumer more than $10^{9} \mathrm{CFU} /$ serving of a probiotic strain as required by recognized standards for probiotic foods. With regard to the organoleptic profile, the process for preparing probiotic table olives allows to obtain a final low-salt product with improved flesh texture and flavors that meets commercial and functional requirements throughout its shelf-life of several months.

\section{CONCLUSIONS}

Technological aspects approached for the development of probiotic table olives demonstrated that the human isolate $L$. paracase IMPC 2.1 is a good example of a probiotic strain suitable for industrial fermentation of de-bittered table olives. In fact, it combines the positive characteristics of a probiotic culture with the efficacy of a starter culture which can control the fermentation process and protect table olives during storage even under adverse conditions of temperature and salt concentration, leading to a final low-salt-probiotic product with functional appeal and high organoleptic quality. "Probiotic table olives" are part of a new probiotic vegetable line of food products which was successfully developed and patented (Lavermicocca et al., 2004) by cooperative actions between research institution and food industry, providing a concrete opportunity to convey in the gastronomy sector probiotic benefits already appreciated by consumers in other market sectors (yogurt and dairy). Probiotic vegetable preparations will have the potential to attract more consumers to functional products including also individuals who are intolerant to milk and its derivatives or require low-cholesterol diets.

\section{ACKNOWLEDGMENTS}

This research was supported by the Italian Ministry of Education, University and Research (art. 12 D.M. 593/2000 - D.D. 3300 22 December 2005 - tema 2) Project "Ortobiotici pugliesi” (D.M. 28830).

F19: survival, ecology and safety in the human intestinal tract - a survey of feeding studies within the PROBDEMO project. Microb. Ecol. Health Dis. S3, 22-26.

D’Arienzo, R., Bozzella, G., Rossi, M., De Bellis, P., Lavermicocca, P., and Sisto, A. (2011). Distinct immunomodulatory properties of Lactobacillus paracasei strains. J. Appl. Microbiol. 111, 1482-1491.

D’Arienzo, R., Maurano, F., Lavermicocca, P., Ricca, E., and Rossi, M. (2009). Modulation of the immune response by probiotic strains in a mouse model of gluten sensitivity. Cytokine 48, 254-259.

Daniel, C., Poiret, S., Goudercourt, D., Dennin, V., Leyer, G., and
Pot, B. (2006). Selecting lactic acid bacteria for their safety and functionality by use of a mouse colitis model. Appl. Environ. Microbiol. 72 , 5799-5805.

De Bellis, P., Valerio, F., Sisto, A., Lonigro, S. L., and Lavermicocca, P. (2010). Probiotic table olives: microbial populations adhering on olive surface in fermentation sets inoculated with the probiotic strain $\mathrm{Lac}$ tobacillus paracasei IMPC 2.1 in an industrial plant. Int. J. Food Microbiol. 140, 6-13.

de Castro, A., Montano, A., Casado, F. J., Sanchez, A. H., and Rejano, L. (2002). Utilization of Enterococcus casselifavus and Lactobacillus pentosus as starter cultures for Spanish-style 
green olive fermentation. Food Microbiol. 19, 637-644.

Delcenserie, V., Martel, D., Lamoureux, M., Amiot, J., Boutin, Y., and Roy, D. (2008). Immunomodulatory effects of probiotics in the intestinal tract. Curr. Issues Mol. Biol. 10, 37-54.

Elo, S., Saxelin, M., and Salminen, S. (1991). Attachment of Lactobacillus case $i$ K strain GG to human colon carcinoma cell line Caco-2: comparison with other dairy strains. Lett. Appl. Microbiol. 13, 154-156.

FAO/WHO. (2001). Health and Nutritional Properties of Probiotics in Food Including Powder Milk with Live Lactic Acid Bacteria. Report on Joint FAO/WHO Expert Consultation on Evaluation of Health and Nutritional Properties of Probiotics in Food Including Powder Milk with Live Lactic Acid Bacteria. Cordoba: FAO/WHO.

FAO/WHO. (2002). Guidelines for the Evaluation of Probiotics in Food. Report of a Joint FAO/WHO Working Group on Drafting Guidelines for the Evaluation of Probiotics in Food. London, ON: FAO/WHO.

Garrido-Fernández, A., FernándezDiaz, M. J., and Adams, M. R. (1997). Table Olives: Production and Processing. London: Chapman \& Hall.

Health Canada. (2009). The Use of Probiotic Microorganisms in Food. Available at: http://www.inspection. gc.ca/english/fssa/labeti/guide/ch8ae. shtml\#a8_7

Lavermicocca, P., Gobbetti, M., Corsetti, A., and Caputo, L. (1998). Characterization of lactic acid bacteria isolated from olive phylloplane and table olive brines. Ital. J. Food Sci. 10, 27-39.

Lavermicocca, P., Lonigro, S. L., Valerio, F., Visconti, A., Vanadia, S., Calabrese, N., Di Venere, D., and Morelli, L. (2004). Process for the preparation of vegetable preserves containing probiotic micro-organisms. Priority date: Italy, 5.10.2004, no MI2004A001887. PCT no WO 2006 / 037517 A1; Italian Patent 001357149 (granted 9.3.2009).

Lavermicocca, P., Lonigro, S. L., Visconti, A., De Angelis, M., Valerio, F., and Morelli, L. (2003). Table olives containing probiotic microorganisms. Priority date: 5.12.2003 no MI2003A002391. European Patent EP1843664 (granted 8.7.09)
Lavermicocca, P., Valerio, F., Lonigro, S. L., De Angelis, M., Morelli, L., Callegari, M. L., Rizzello, C. G., and Visconti, A. (2005). Study of adhesion and survival of Lactobacilli and Bifidobacteria on table olives with the aim of formulating a new probiotic food. Appl. Environ. Microbiol. 71, 4233-4240.

Lavermicocca, P., Valerio, F., Lonigro, S. L., Di Leo, A., and Visconti, A. (2008). Antagonistic activity of potential probiotic lactobacilli against the ureolytic pathogen Yersinia enterocolitica. Curr. Microbiol. 56, 175-181.

Lebeer, S., Vanderleyden, J., and De Keersmaecker, S. C. J. (2008). Genes and molecules of lactobacilli supporting probiotic action. Microbiol. Mol. Biol. Rev. 72, 728-764.

Maki, M., Mustalahti, K., Kokkonen, J., Kulmala, P., Haapalahti, M., Karttunen, T., Ilonen, J., Laurila, K., Dahlbom, I., Hansson, T., Höpfl, P., and Knip, M. (2003). Prevalence of Celiac disease among children in Finland. N. Engl. J. Med. 348, 2517-2524. Maukonen, J., Alakomi, H. L., Nohynek, L., Hallamaa, K., Leppämäki, S., Mättö, J., and Saarela, M. (2006). Suitability of the fluorescent techniques for the enumeration of probiotic bacteria in commercial non-dairy drinks and in pharmaceutical products. Food Res. Int. 39, 22-32.

Ohlson, K., Björneholm, S., Fonden, R., and Svensson, U. (2002). Lactobacillus F19 - A probiotic strain suitable for consumer products. Microb. Ecol. Health Dis. S3, 27-32.

Panagou, E. Z., Tassou, C. C., and Katsaboxakis, C. Z. (2003). Induced lactic acid fermentation of untreated green olives of the Conservolea cultivar by Lactobacillus pentosus. J. Sci. Food Agric. 83, 667-674.

Panagou, E. Z., Tassou, C. C., Saravanos, E. K. A., and Nychas, G. J. (2007). Application of neural networks to simulate the growth profile of lactic acid bacteria in green olive fermentation. J. Food Prot. 70, 1909-1916.

Reid, G., Howard, J., and Gan, B. S. (2001). Can bacterial interference prevent infection? Trends Microbiol. 9, 424-428.
Reid, G., Sanders, M. E., Gaskins, H. R., Gibson, G. R., Mercenier, A., Rastall, R., Roberfroid, M., Rowland, I., Cherbut, C., and Klaenhammer, T. R. (2003). New scientific paradigms for probiotics and prebiotics. J. Clin. Gastroenterol. 37, 105-118.

Riezzo, G., Orlando, A., D’Attoma, B., Guerra, V., Valerio, F., Lavermicocca, P., and De Candia, S. (2012). Randomised clinical trial: efficacy of Lactobacillus paracaseienriched artichokes in the treatment of patients with functional constipation - a double-blind, controlled, crossover study. Aliment. Pharmacol. Ther doi: 10.1111/j.1365-2036.2011. 04970.x.

Sánchez, A. H., Rejano, L., Montaño, A., and de Castro, A. (2001). Utilisation at high $\mathrm{pH}$ of starter cultures of lactobacilli for Spanish-style green olive fermentation. Int. J. Food Microbiol. 67, 115-122.

Senger, S., Maurano, F., Mazzeo, M. F., Gaita, M., Fierro, O., David, C. S., Troncone, R., Auricchio, S., Siciliano, R. A., and Rossi, M. (2005). Identification of immunodominant epitopes of alpha-gliadin in HLA-DQ8 transgenic mice following oral immunization. J. Immunol. 175, 8087-8095.

Servin, A. L., and Coconnier, M. H (2003). Adhesion of probiotic strains to the intestinal mucosa and interaction with pathogens. Best Pract. Res. Clin. Gastroenterol. 17, 741-754.

Sisto, A., De Bellis, P., Visconti, A., Morelli, L., and Lavermicocca, P. (2009). Development of a PCR assay for the strain-specific identification of the probiotic strain Lactobacillus paracasei IMPC 2.1. Int. J. Food Microbiol. 136, 59-65.

Valerio, F., De Bellis, P., Lonigro, S. L., Morelli, L., Visconti, A., and Lavermicocca, P. (2006). In vitro and in vivo survival and transit tolerance of potentially probiotic strains carried by artichokes in the gastrointestinal tract. Appl. Environ. Microbiol. 72, 3042-3045.

Valerio, F., de Candia, S., Lonigro, S. L., Russo, F., Riezzo, G., Orlando, A., De Bellis, P., Sisto, A., and Lavermicocca, P. (2011). Role of the probiotic strain Lactobacillus paracasei LMGP22043 carried by artichokes in influencing faecal bacteria and biochemical parameters in human subjects. $J$ Appl. Microbiol. 111, 155-164.

Valerio, F., Russo, F., de Candia, S., Riezzo, G., Orlando, A., Lonigro, S. L., and Lavermicocca, P. (2010). Effects of probiotic Lactobacillus paracaseienriched artichokes on constipated patients: a pilot study. J. Clin. Gas troenterol. 44(Suppl. 1), S49-S53.

Van den Berg, D. J. C., Smits, A., Pot, B., Ledeboer, A. M., Kesters, K., Verbakel, J. M. A., and Verrips, C. T. (1993). Isolation, screening and identification of lactic acid bacteria from traditional food fermentation processes and culture collections. Food Biotechnol. 7, 189-205.

Vega Leal-Sánchez, M., Ruiz-Barba, J. L., Sánchez, A. H., Rejano, L., JiménezDíaz, R., and Garrido, A. (2003). Fermentation profile and optimization of green olive fermentation using Lactobacillus plantarum LPCO10 as a starter culture. Food Microbiol. 20, 421-430.

Yan, F., and Polk, D. B. (2011). Probiotics and immune health. Curr. Opin. Gastroenterol. 27, 496-501.

Conflict of Interest Statement: The authors declare that the research was conducted in the absence of any commercial or financial relationships that could be construed as a potential conflict of interest.

Received: 25 January 2012; paper pending published: 14 February 2012; accepted: 19April 2012; published online: 08 May 2012.

Citation: Sisto $A$ and Lavermicocca $P$ (2012) Suitability of a probiotic Lactobacillus paracasei strain as a starter culture in olive fermentation and development of the innovative patented product "probiotic table olives". Front. Microbio. 3:174. doi: 10.3389/fmicb.2012.00174 This article was submitted to Frontiers in Food Microbiology, a specialty of Frontiers in Microbiology.

Copyright (c) 2012 Sisto and Lavermicocca. This is an open-access article distributed under the terms of the Creative Commons Attribution Non Commercial License, which permits noncommercial use, distribution, and reproduction in other forums, provided the original authors and source are credited. 